\title{
An imaging-based approach predicts clinical outcomes in prostate cancer through a novel support vector machine classification
}

\author{
Yu-Dong Zhang ${ }^{1}$, Jing Wang ${ }^{2}$, Chen-Jiang Wu${ }^{1}$, Mei-Ling Bao ${ }^{3}$, Hai $\mathrm{Li}^{3}$, Xiao-Ning \\ Wang $^{1}$, Jun TaO ${ }^{4}$ and Hai-Bin Shi ${ }^{1}$ \\ ${ }^{1}$ Department of Radiology, the First Affiliated Hospital with Nanjing Medical University, Nanjing, China \\ ${ }^{2}$ Center for Medical Device Evaluation, CFDA, Beijing, China \\ ${ }^{3}$ Department of Pathology, the First Affiliated Hospital with Nanjing Medical University, Nanjing, China \\ ${ }^{4}$ Department of Urology, the First Affiliated Hospital with Nanjing Medical University, Nanjing, China \\ Correspondence to: Hai-Bin Shi, email: njmu_shb@163.com \\ Keywords: prostate cancer; biochemical recurrence; MRI; radical prostatectomy; support vector machine \\ Received: April 27, 2016 \\ Accepted: August 11, 2016 \\ Published: August 15, 2016
}

\section{ABSTRACT}

Preoperatively predict the probability of Prostate cancer (PCa) biochemical recurrence (BCR) is of definite clinical relevance. The purpose of this study was to develop an imaging-based approach in the prediction of 3-years BCR through a novel support vector machine (SVM) classification. We collected clinicopathologic and MR imaging datasets in 205 patients pathologically confirmed PCa after radical prostatectomy. Univariable and multivariable analyses were used to assess the association between MR findings and 3-years BCR, and modeled the imaging variables and follow-up data to predict 3-year PCa BCR using SVM analysis. The performance of SVM was compared with conventional Logistic regression (LR) and D'Amico risk stratification scheme by area under the receiver operating characteristic curve (Az) analysis. We found that SVM had significantly higher Az (0.959 vs. 0.886; $p=$ $0.007)$, sensitivity $(93.3 \%$ vs. $83.3 \% ; p=0.025)$, specificity $(91.7 \%$ vs. $77.2 \% ; p=$ $0.009)$ and accuracy $(92.2 \%$ vs. $79.0 \% ; p=0.006)$ than LR analysis. Performance of popularized D'Amico scheme was effectively improved by adding MRI-derived variables (Az: 0.970 vs. $0.859, p<0.001$; sensitivity: $91.7 \%$ vs. $86.7 \%, p=0.031$; specificity: $94.5 \%$ vs. $78.6 \%, p=0.001$; and accuracy: $93.7 \%$ vs. $81.0 \%, p=0.007$ ). Additionally, beside pathological Gleason score (hazard ratio $[H R]=1.560, p=$ $0.008)$, surgical-T3b $(H R=4.525, p<0.001)$ and positive surgical margin $(H R=$ 1.314, $p=0.007)$, apparent diffusion coefficient $(H R=0.149, p=0.035)$ was the only independent imaging predictor of time to PSA failure. Therefore, We concluded that imaging-based approach using SVM was superior to LR analysis in predicting PCa outcome. Adding MR variables improved the performance of D'Amico scheme.

\section{INTRODUCTION}

Radical prostatectomy (RP) is an effective form of local therapy for prostate cancer (PCa) [1,2]. However, there is still approximately one quarter of patients undergoing this curative surgery will have a biochemical recurrence (BCR) or "prostate-specific antigen (PSA) recurrence" [1-5]. Preoperatively predict the probability of BCR is of definite clinical relevance. Several preoperative nomograms, i.e., the Stephenson nomogram [6], the
D'Amico risk stratification scheme [7], and the University of California, San Francisco, Cancer of the Prostate Risk Assessment (CAPRA) score [8], have been developed in the urologic community to predict the probability of BCR within 3-5 or 10 years of treatment. Although these nomograms have been internationally validated, unfortunately, only a few of them have predicted the probability of 5-year BCR with more than $70 \%$ accuracy [9-11]. Therefore, efforts to improve existing outcome prediction tools in $\mathrm{PCa}$ are always encouraged. 
In the past decades, multi-parametric MRI (mp$\mathrm{MRI}$ ), i.e., T1-weighted imaging ( $\left.\mathrm{T}_{1} \mathrm{WI}\right), \mathrm{T} 2$-weighted imaging $\left(\mathrm{T}_{2} \mathrm{WI}\right)$, diffusion weighted imaging (DWI), dynamic contrast-enhanced (DCE), and MR spectroscopic imaging, has been investigated as promising way not only for imaging, detecting and staging the localized cancer, but also for risk stratification, image guidance for biopsy, surgery and focal therapy [12-18]. However, the clinical application of mp-MRI can be challenged by large amount of image data in each patient. Additionally, as without standardized ways, the image interpretation can be subjective, which depends on radiologists' experience, level of local medical culture and personal preference, thereby limiting the diagnostic accuracy and reproducibility in PCa [19]. Recently, automated cancer detecting and classification based on preoperative mpMRI through a novel support vector machine (SVM) analysis has been an ongoing interest. SVM is one of the best-known classification techniques and usually provides the best classification for computer-aided detection in radiology [20]. Recent technical developments in SVM by expending advanced algorithms (e.g., principal component analysis [21, 22], particle swarm optimization [23] and cross-validation schemes) have produced encouraging results regarding solving or classifying pattern recognition problems [24]. The SVM analysis has the ability of reducing false positives in the determination of abnormal lesions in brain [24, 25], breast [26] and prostate [20], showing high accuracy, elegant mathematical tractability and direct geometric interpretation. Although the application of SVM to the diagnosis of PCa has validity in research, a more meaningful clinical application of it is in helping the identification of predictors of outcome $[9,27,28]$. These could help direct, to more highrisk individuals, the early implementation of targeted interventions that have been shown to reduce relapse rates, such as optimized neo-adjuvant therapy, resulting in better clinical outcomes. However, there is not yet a single, widely accepted algorithm for establishing that predictive nomogram by mp-MRI.

The purpose of this study was therefore: (1) to develop an imaging-based nomogram to predict 3-years BCR in patients with localized PCa after RP using SVM analysis; (2) to determine whether SVM could be better than a conventional logistic regression (LR) analysis to improve performance ability; (3) to evaluate whether adding MRI-derived variables can effectively complement the preoperative clinico-pathologic predictors in patient outcome predicting.

\section{RESULTS}

\section{Clinical characteristics}

Clinical, histologic variables and MR findings for the study cohort (205 patients) are depicted in Table 1 . The median age was $68 \mathrm{yr}$ (interquartile range [IQR]: 62- 73 ys), and median serum PSA was $13.1 \mathrm{ng} / \mathrm{ml}$ (IQR: 7.917.7). As of January 2016, 61 (29.7\%) of the 205 patients in the study had a biochemical recurrence. The median (range) follow-up for all patients was 43.8 (2-60) months; it was 47.3 (37-60) months for those with no evidence of recurrence and 20.1 (2-41) months for those with recurrence. Of the 205 prostatectomy confirmed patients, total 409 lesions were detected at histological findings, whereas total 263 lesions were detected at MR findings. Tumor in $178(86.8 \%)$ patients originated in $\mathrm{PZ}$ and 27 $(13.2 \%)$ originated in $\mathrm{TZ}$ on section histology.

\section{Performance of the predictive models}

Table 2 shows the results of the multiple LR analysis. The estimate probabilities $(\mathrm{Pi})$ of PCa BCR after RP is: $P i=1 /(1+\exp (-0.421+1.226 *$ age $+1.211 *$
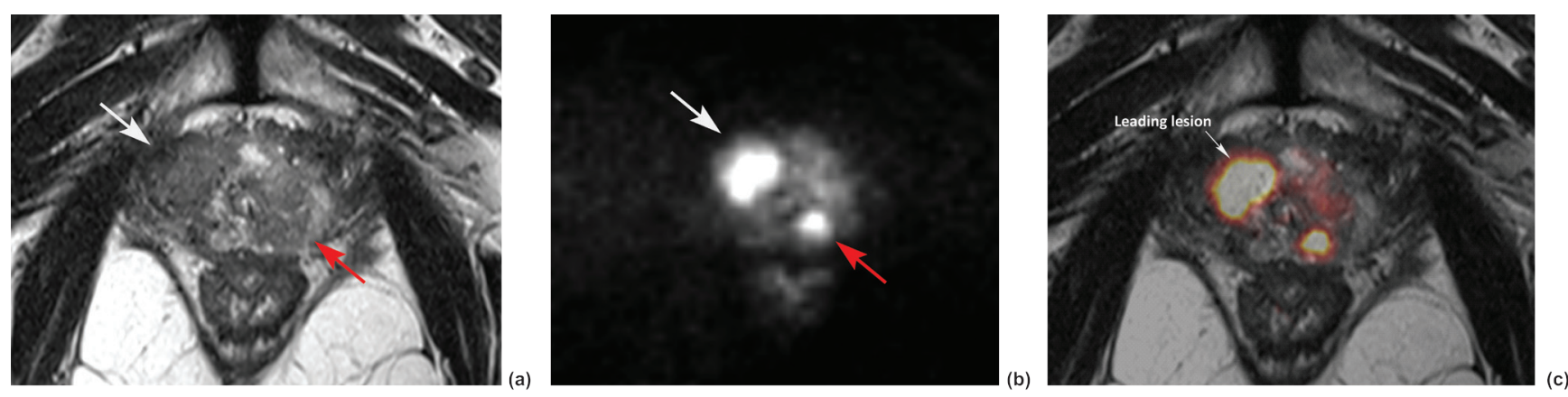

Figure 1: MR images of a representative case to indicate the imaging registration, lesion identification and region of interesting drawing. A 69-year-old man presented with two solid tumor foci in right peripheral zone (PZ; write arrow) (pathological Gleason score 4+4, pT3a) and left PZ (pathological Gleason score 4+3), respectively. The two lesions were characterized with hypo-signal intensity (SI) on T2-weighted imaging (T2WI; a), and hyper-SI on diffusion-weighted imaging (DWI; b = $1000 \mathrm{~s} / \mathrm{mm}^{2}$ ). Registration and fusion of images between T2WI and DWI was performed using a. DICOM-tag metrics c., showing clearly the lesion boundaries. Because the lesion in right $\mathrm{TZ}$ had larger size, and an infiltration of the peri-prostatic fat (extracapsular extension) was suspected, it was selected as leading lesion for further quantitative measure. 


\begin{tabular}{|c|c|c|}
\hline Variables & Mean (SD) or n (\%) & \\
\hline \multicolumn{3}{|l|}{ Preoperative Characteristics } \\
\hline \multicolumn{3}{|l|}{ Age, $\mathrm{n}(\%)$} \\
\hline$\leq 60$ ys & \multicolumn{2}{|l|}{$40(19.5 \%)$} \\
\hline$>60$ ys & \multicolumn{2}{|l|}{$165(80.5 \%)$} \\
\hline \multicolumn{3}{|l|}{ Clinical stage, $\mathrm{n}(\%)$} \\
\hline$<\mathrm{T} 3$ & \multicolumn{2}{|l|}{$118(57.6 \%)$} \\
\hline$\geq \mathrm{T} 3$ & \multicolumn{2}{|l|}{$87(42.4 \%)$} \\
\hline \multicolumn{3}{|l|}{ Preoperative PSA level, n (\%) } \\
\hline $0-10 \mathrm{ng} / \mathrm{ml}$ & \multicolumn{2}{|l|}{$81(39.5 \%)$} \\
\hline $10-20 \mathrm{ng} / \mathrm{ml}$ & \multicolumn{2}{|l|}{$100(48.8 \%)$} \\
\hline$>20 \mathrm{ng} / \mathrm{ml}$ & \multicolumn{2}{|l|}{$24(11.7 \%)$} \\
\hline \multicolumn{3}{|l|}{ Biopsy Gleason score, n (\%) } \\
\hline$\leq 3+3$ & \multicolumn{2}{|l|}{$88(42.9 \%)$} \\
\hline $3+4$ & \multicolumn{2}{|l|}{$55(26.8 \%)$} \\
\hline $4+3$ & \multicolumn{2}{|l|}{$44(21.5 \%)$} \\
\hline$\geq 4+4$ & \multicolumn{2}{|l|}{$18(8.8 \%)$} \\
\hline \multicolumn{3}{|l|}{ Preoperative MR findings } \\
\hline \multicolumn{3}{|l|}{ Lesion Location, n (\%) } \\
\hline $\mathrm{PZ}$ & \multicolumn{2}{|l|}{$178(86.8 \%)$} \\
\hline $\mathrm{TZ}$ & \multicolumn{2}{|l|}{$27(13.2 \%)$} \\
\hline \multicolumn{3}{|l|}{ DCE type } \\
\hline Type $1+2$ & \multicolumn{2}{|l|}{$86(41.9 \%)$} \\
\hline Type 3 & \multicolumn{2}{|l|}{$119(58.0 \%)$} \\
\hline $\operatorname{ADCs}\left(\times 10^{-3} \mathrm{~mm}^{2} / \mathrm{s}\right)$, Mean $(\mathrm{SD})$ & \multicolumn{2}{|l|}{$1.06(0.19)$} \\
\hline Max diameter $(\mathrm{cm})$, Mean $(\mathrm{SD})$ & $1.8(0.8)$ & \\
\hline MR-detected T stage & & \\
\hline$<\mathrm{T} 3 \mathrm{a}$ & $114(55.6 \%)$ & \\
\hline T3a (ECE) & $56(27.3 \%)$ & \\
\hline T3b (SVI) & $35(17.1 \%)$ & \\
\hline Postoperative Characteristics & & \\
\hline Pathological Gleason score, $\mathrm{n}(\%)$ & & \\
\hline$\leq 3+3$ & $48(23.4 \%)$ & \\
\hline $3+4$ & $75(36.6 \%)$ & \\
\hline $4+3$ & $48(23.4 \%)$ & \\
\hline$\geq 4+4$ & $34(16.6 \%)$ & \\
\hline Surgical tumor volume, Mean (SD & & \\
\hline Volume, $\mathrm{cm}^{3}$ & $5.4(9.1)$ & \\
\hline Volume percentage, $\%$ & $14.5(14.6)$ & \\
\hline Surgical ECE, n (\%) & & \\
\hline Absent & $133(64.9 \%)$ & \\
\hline Present & $72(35.1 \%)$ & \\
\hline Surgical SVI, n (\%) & & \\
\hline Absent & $166(81.0 \%)$ & \\
\hline Present & $39(19.0 \%)$ & \\
\hline Cancer invasion on surgical margi & & \\
\hline Negative & $154(75.1 \%)$ & \\
\hline Positive & $51(24.9 \%)$ & \\
\hline
\end{tabular}




\begin{tabular}{|l|l|l|l|l|l|l|l|}
\hline Table 2: Logistic regression (LR) model with multiple variables \\
\hline \multirow{2}{*}{ Variable } & Coefficient B & S.E. & Wald & $\boldsymbol{p}$ & \multirow{2}{*}{$\boldsymbol{E}(\mathbf{B})$} & $\mathbf{9 5 \%}$ C.I. for EXP(B) \\
\cline { 7 - 9 } & & & & & & Lower & Upper \\
\hline DCE type & 0.407 & 0.443 & 0.743 & 0.359 & 1.502 & 0.630 & 3.579 \\
\hline ADCs & -4.196 & 1.346 & 9.722 & $0.002^{*}$ & 0.015 & 0.001 & 0.210 \\
\hline Location & -0.150 & 0.567 & 0.069 & 0.792 & 0.861 & 0.283 & 2.618 \\
\hline MR visible & 0.645 & 1.172 & 0.303 & 0.582 & 1.906 & 0.191 & 18.974 \\
\hline PI-RADS & 0.266 & 0.403 & 0.434 & 0.510 & 1.304 & 0.592 & 2.874 \\
\hline Dmax & -0.170 & 0.280 & 0.369 & 0.543 & 0.844 & 0.488 & 1.459 \\
\hline MR T-stage & 1.211 & 0.299 & 16.397 & $0.001^{*}$ & 3.358 & 1.868 & 6.037 \\
\hline Age & 1.226 & 0.643 & 3.637 & 0.057 & 3.406 & 0.967 & 12.002 \\
\hline Constant & -0.421 & 2.259 & 0.035 & 0.852 & 0.656 & & \\
\hline C.I. = Confidence Interval, S.E. = Standard Error. PI-RADS = Prostate Imaging and Reporting and Data System. \\
\hline
\end{tabular}

Table 3: Predictabilities of LR, ANN and SVM models in prediction of PCa BCR

\begin{tabular}{|c|c|c|c|c|c|}
\hline Model & $\mathbf{A z}$ & SEN, \% & SPE, \% & $\mathrm{ACC}, \%$ & cutoff value \\
\hline $\mathrm{LR}_{\mathrm{MR}}$ & $0.886(0.834-0.926)$ & $83.3(71.5-91.7)$ & $77.2(69.5-83.8)$ & $79.0(70.1-86.2)$ & $\mathrm{Pi}>0.41$ \\
\hline $\mathrm{SVM}_{\mathrm{MR}}$ & $0.959(0.922-0.982)$ & $93.3(83.8-98.1)$ & $91.7(86.0-95.6)$ & $92.2(85.3-96.3)$ & $\mathrm{Pi}>0.44$ \\
\hline $\mathrm{SVM}_{\mathrm{D}^{\prime} \mathrm{An}^{\prime}}$ & $0.859(0.804-0.903)$ & $86.7(75.4-94.0)$ & $78.6(71.0-85.0)$ & $81.0(72.3-87.7)$ & $\mathrm{Pi}>0.41$ \\
\hline $\mathrm{SVM}_{\mathrm{D}^{\prime} \text { Amico }+\mathrm{MR}}$ & $0.970(0.936-0.988)$ & 91.7 (81.6- 97.2) & $94.5(89.4-97.6)$ & $93.7(87.1-97.4)$ & $\mathrm{Pi}>0.40$ \\
\hline
\end{tabular}

\begin{tabular}{|c|c|c|c|c|c|}
\hline \multirow[b]{2}{*}{ Variable } & \multicolumn{2}{|c|}{ Kaplan-Meier analysis } & \multicolumn{3}{|c|}{ multivariate Cox analysis } \\
\hline & $\begin{array}{c}\text { Log-rank } \\
\text { (Mantel-Cox) }\end{array}$ & $p$ & B & HR $(95 \%$ CI) & $p$ \\
\hline \multicolumn{6}{|c|}{ preoperative clinic-pathologic variables } \\
\hline$\overline{\text { Age }}$ & 8.691 & 0.003 & & & \\
\hline PSA level & 108.833 & $<0.001$ & & & \\
\hline Biopsy GS & 79.987 & $<0.001$ & & & \\
\hline Clinical stage & 19.963 & $<0.001$ & & & \\
\hline \multicolumn{6}{|l|}{ Preoperative MR variables } \\
\hline Tumor location & 0.445 & 0.505 & & & \\
\hline MR-visible & 10.558 & 0.001 & & & \\
\hline PI-RADS & 18.366 & $<0.001$ & & & \\
\hline Dmax & 13.663 & $<0.001$ & & & \\
\hline DCE type & 14.015 & $<0.001$ & & & \\
\hline $\mathrm{ADCs}$ & 42.122 & $<0.001$ & -1.904 & $0.149(0.025-0.873)$ & $0.035^{\dagger}$ \\
\hline MR T stage & 90.668 & $<0.001$ & & & \\
\hline \multicolumn{6}{|l|}{ postoperative variables } \\
\hline Volume & 12.153 & $<0.001$ & & & \\
\hline Volume percentage & 12.938 & $<0.001$ & & & \\
\hline Pathological GS & 71.625 & $<0.001$ & 0.445 & $1.560(1.124-2.166)$ & $0.008^{\dagger}$ \\
\hline Surgical T-stage & 128.576 & $<0.001$ & 1.510 & $4.525(2.441-8.386)$ & $<0.001$ \\
\hline Surgical margin & 70.056 & $<0.001$ & 0.839 & $2.314(1.260-4.251)$ & $0.007^{\dagger}$ \\
\hline Perineural invasion & 11.632 & 0.001 & & & \\
\hline
\end{tabular}


MR T-stage $-0.170 *$ Dmax $+0.645 *$ MR visible -0.150 * location - 4.196* ADCs + 0.407* DCE type + 0.266* PI-RADS)). It shows that tumor ADCs and MR T-stage are significantly associated with 3 -years BCR $(p<0.05)$.

Figure 3 shows the ROC curves for the four risk predictive models. The area under the ROC curve values (Az) for the model of $\mathrm{LR}_{\mathrm{MR}}, \mathrm{SVM}_{\mathrm{MR}}, \mathrm{SVM}_{\mathrm{D} \text { 'Amico, }}$ and $\mathrm{SVM}_{\text {D'Amico+MR }}$ was $0.886,0.959,0.859$, and 0.970 , respectively. Pairwise comparison of the ROC curves demonstrated that there was statistical difference between $\mathrm{LR}_{\mathrm{MR}}$ and $\mathrm{SVM}_{\mathrm{MR}}$ (Figure 4a; $p=0.007$ ), and between $\mathrm{SVM}_{\mathrm{D} \text { 'Amico }}$ and $\mathrm{SVM}_{\mathrm{D} \text { 'Amico+MR }}$ (Figure 4b; $p<0.001$ ). Using the optimal cutoff values obtained from ROC analysis (Table 3), $\mathrm{SVM}_{\mathrm{MR}}$ had significantly higher diagnostic SEN $(p=0.025), \operatorname{SPE}(p=0.009)$ and ACC $(p=0.006)$ than $\mathrm{LR}_{\mathrm{MR}}$ in 3 -years $\mathrm{BCR}$ predicting. $\mathrm{SVM}_{\mathrm{D} \text { 'Amico+MR }}$ had significantly higher diagnostic SEN $(p=0.031), \operatorname{SPE}(p=0.001)$ and $\operatorname{ACC}(p=0.007)$ than $\mathrm{SVM}_{\mathrm{D}^{\prime} \text { Amico }}$ The difference of Az, SEN, SPE and ACC between $\mathrm{SVM}_{\mathrm{MR}}$ and $\mathrm{SVM}_{\mathrm{D}^{\prime} \text { Amico+MR }}$ was insignificant (all $p>0.05$ ). Figure 4 shows the predicted BCR-free survival curves of 205 patients by $\mathrm{LR}_{\mathrm{MR}}, \mathrm{SVM}_{\mathrm{MR}}, \mathrm{SVM}_{\mathrm{D} \text { 'Amico }}$ and $\mathrm{SVM}_{\mathrm{D} \text { 'Amico+MR }}$ model, respectively, which were compared to patients' true survival curve. It shows that the survival functions constructed by $\mathrm{SVM}_{\mathrm{MR}}$ and $\mathrm{SVM}_{\mathrm{D} \text { 'Amico+MR }}$ have relatively smaller bias than $\mathrm{LR}_{\mathrm{MR}}$ and $\mathrm{SVM}_{\mathrm{D}^{\prime} \text { 'Amico }}$.

Among all the preoperative clinic-pathologic variables (age, biopsy GS, clinical TNM stage, and baseline PSA), the MR variables (tumor location, MRvisible, Dmax, DCE type, ADCs, PI-RADS score, and MR T-stage), and the postoperative variables (tumor volume, volume percentage, pathological GS, pathological T-stage, cancer invasion on surgical margin, and perineural invasion), the Cox regression multivariate analysis indicated that only the tumor ADCs (HR = $0.149 ; p=0.035)$, pathological $\mathrm{GS}(\mathrm{HR}=1.560 ; p=$ $0.008)$, positive surgical margin $(\mathrm{HR}=2.314 ; p=0.007)$, and surgical SVI $(\mathrm{HR}=4.525 ; p<0.001)$ were the independent risk predictors of time to PSA failure (Table 4). Using the optimal cutoff values for ADCs obtained from ROC analysis $\left(<1.03 \times 10^{-3} \mathrm{~mm}^{2} / \mathrm{s}\right)$, The overall accuracy of ADCs to differentiate low-intermediate grade (pathological GS $\leq 3+4$ ) from high-grade GS > 3+4) PCa is $64.9 \%$. The overall accuracy of MR to determine ECE and SVI is $81.9 \%$ and $95.1 \%$, respectively.
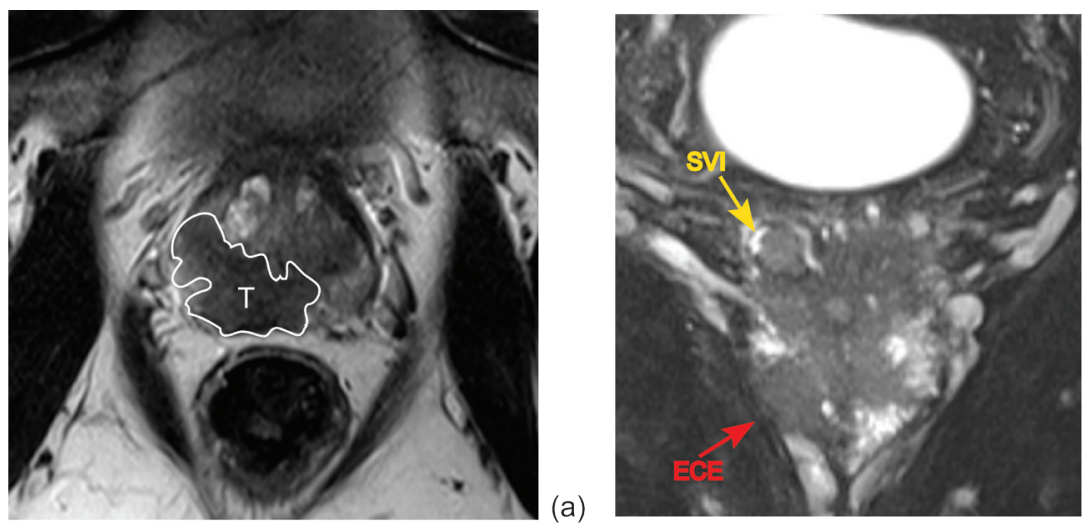

(b)

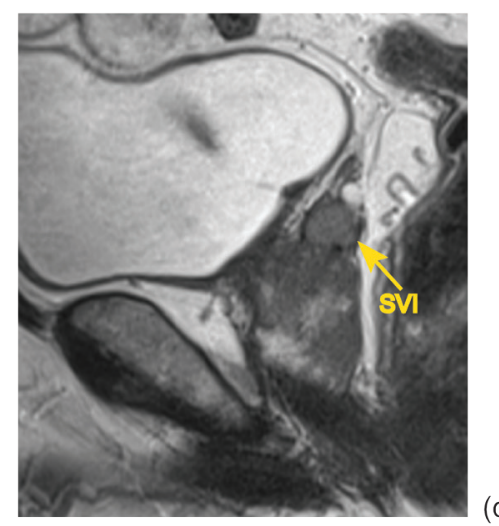

(c)
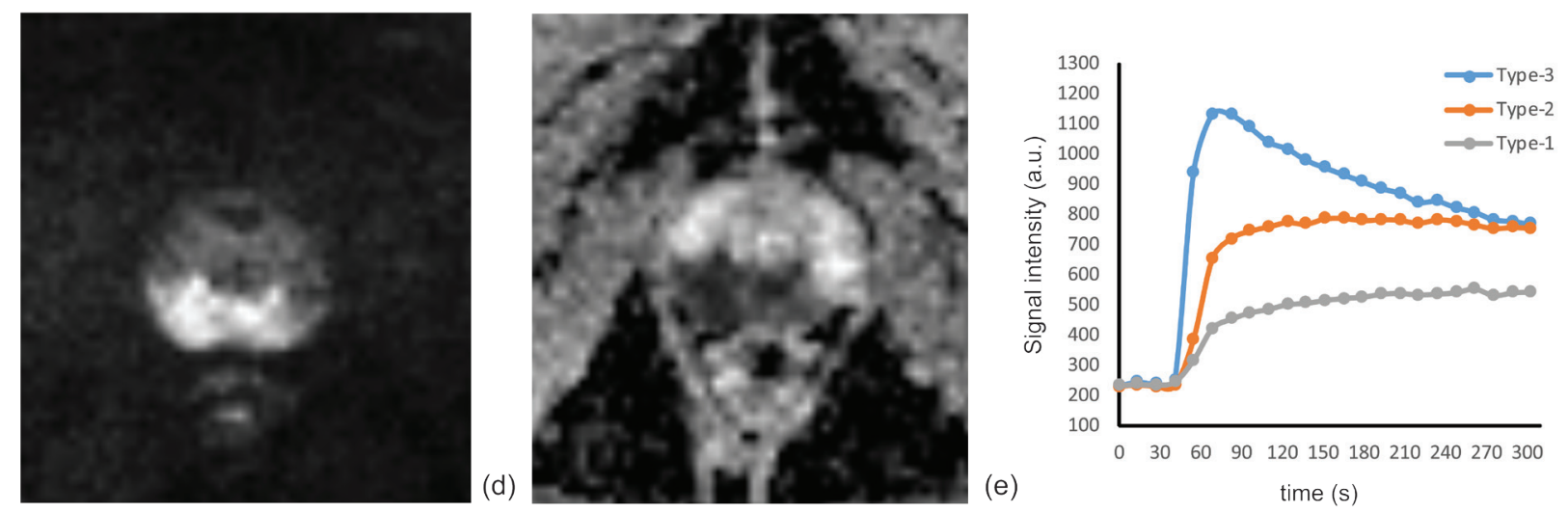

(g)

Figure 2: A multi-parametric prostate MRI in a 68-year-old man (PSA of $34.3 \mathrm{ng} / \mathrm{ml}$, biopsy Gleason score 4+3 and stage T3b) to show the metrics for imaging interpretation. a. Tumor featured with decreased SI on axial $\mathrm{T}_{2} \mathrm{WI}$, the location was defined at PZ (white line). b. ECE (red arrow) and seminal vesicle invasion (SVI; yellow arrow) were notified on coronal $\mathrm{T}_{2} \mathrm{WI}$ with fat suppression. c. Tumor with SVI was confirmed on sagittal $\mathrm{T}_{2} \mathrm{WI}$ (yellow arrow). d. Tumor was characteristic with hyper-SI on DWI $(\mathrm{b}=$ $1000 \mathrm{~s} / \mathrm{mm}^{2}$ ) and decreased ADC e. f. Schematic diagram shows three-type DCE curves, for this patient, tumor was defined as type-3 DCE curve (blue color). Histopathologic results showed a pathological Gleason core $4+4$ and surgical SVI in this patient, and PSA failure was determined on 16 months after the prostatectomy treatment. 


\section{DISCUSSION}

In this study, we demonstrated that the combination of clinicopathologic and imaging variables can effectively contribute to the prediction of clinical outcome in patients with localized PCa before the surgical treatment. The nomogram constructed by SVM was significantly better than LR analysis. Additionally, the performance of popularized D'Amico scheme can be effectively improved by adding mp-MR imaging markers, suggesting this approach could be an optimal tool for initial evaluation before a curative attempt in patients with localized PCa.

The classification methods applied to pelvic MR image data was initially investigated in several studies for automated detection of malignancy [29-31]. Poulakis devised an ANN model with the input variables of MR findings, pretreatment PSA, clinical TNM stage and biopsy GS to predict PCa recurrence in 210 clinically localized PCa [9]. They found the new nomogram can produce higher sensitivity (91\%) and specificity (88\%) than conventional LR analysis to preoperatively predict 5-year PSA failure. Fuchsjäger et al. [32] explored an integrated Cox model based on a seven-point scale MR scoring system and clinical variables to preoperatively predict 5- and 10-year BCR after RP. And recently, Park et al. [33, 34] demonstrated that tumor ADCs derived from DWI and new PI-RADS v2 score was better than preoperative PSA, biopsy GS and surgical variables in the
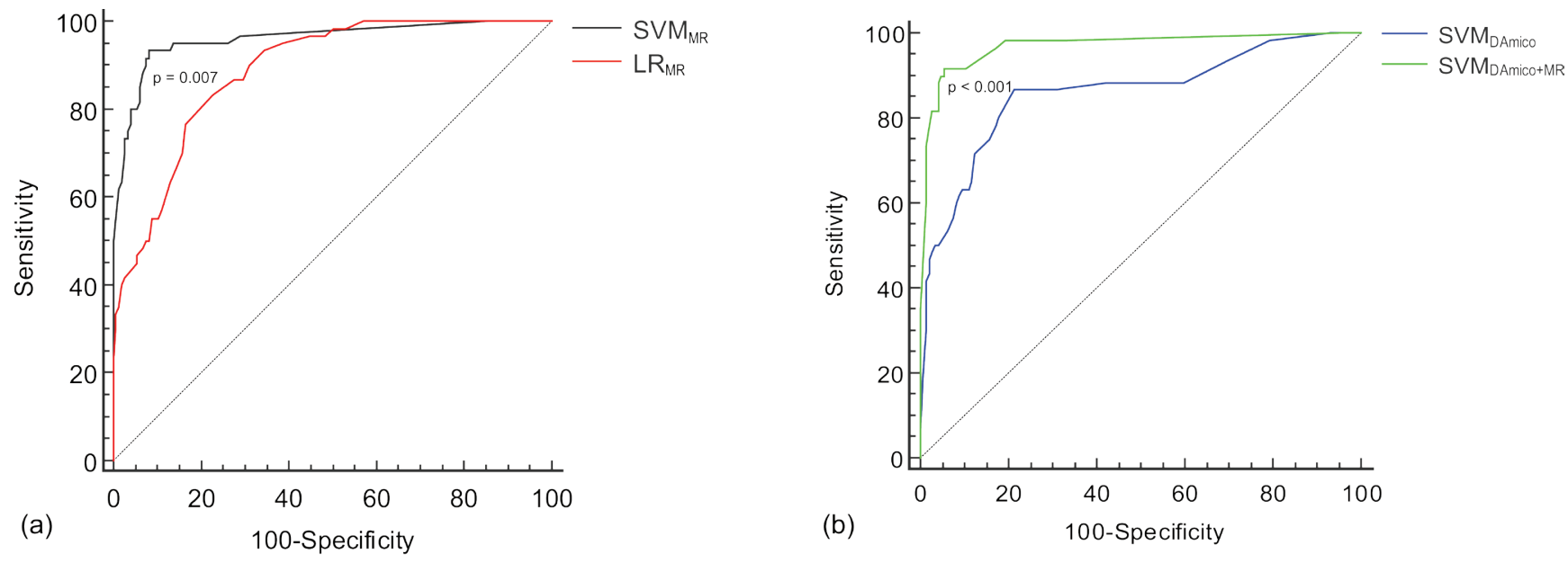

Figure 3: the comparison of ROC curves among four risk predictive models constructed with different classification methods and input variables. $a$. with the same $M R$ input variables, the model constructed by support vector machine $\left(\mathrm{SVM}_{\mathrm{MR}}\right)$ has significantly higher area under the ROC curve value $(A z=0.959)$ than the model of logistic regression $(A z=0.886, p=0.007)$. $\mathbf{b}$. using the same SVM analysis, the model combining MR and DA'mico variables has significantly higher Az (0.970) than the model using sole DA'mico variables $(\mathrm{Az}=0.859 ; p<0.001)$.
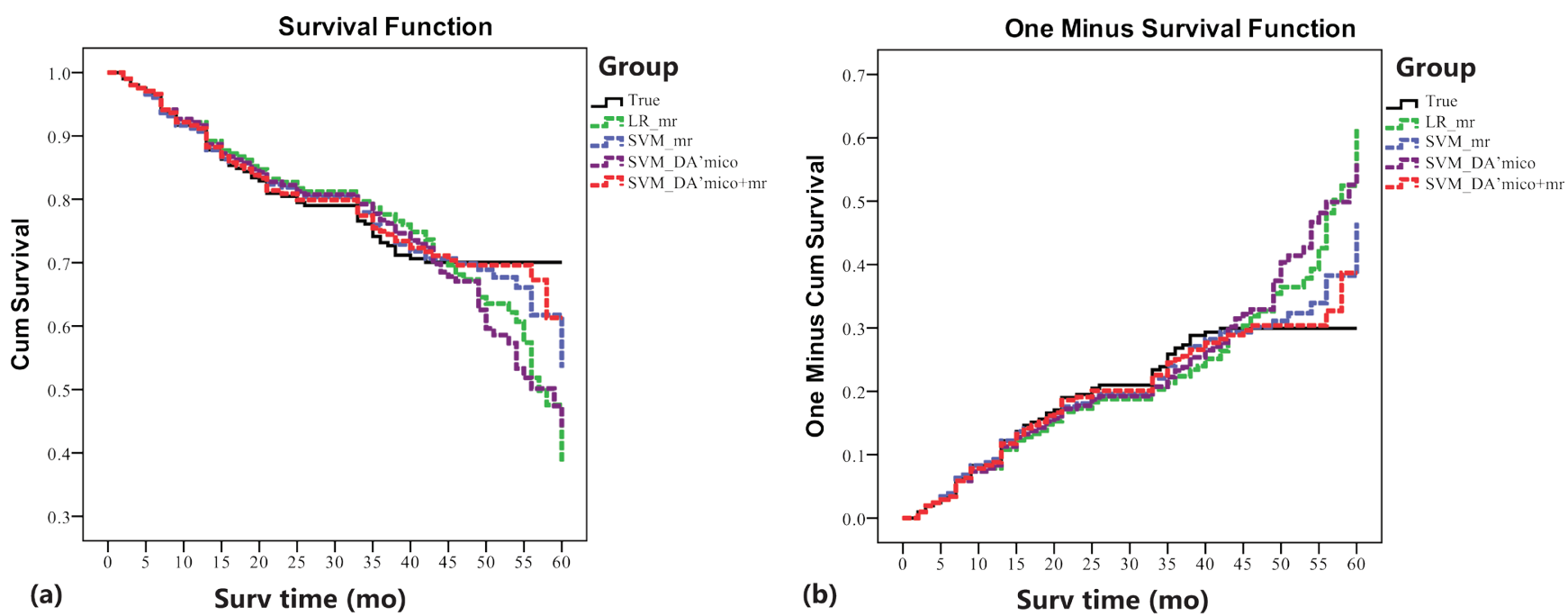

Figure 4: Predicted BCR-free Kaplan-Meier curves of 205 patients after radical prostatectomy by four constructed models. The curves are stratified by: patients' true Kaplan-Meier curve (black), and predicted Kaplan-Meier curves by $\mathrm{LR}_{\mathrm{MR}}$ (green), $\mathrm{SVM}_{\mathrm{MR}}$ (blue), $\mathrm{SVM}_{\mathrm{D}^{\prime} \mathrm{Amico}}$ (brown) and $\mathrm{SVM}_{\mathrm{D}^{\prime} A \operatorname{Amico}+\mathrm{MR}}$ model (red), respectively. It shows that the Kaplan-Meier functions constructed by $\mathrm{SVM}_{\mathrm{MR}}$ and $\mathrm{SVM}_{\mathrm{D}^{\prime} \mathrm{Amico}+\mathrm{MR}}$ have relatively smaller bias than these of $\mathrm{LR}_{\mathrm{MR}}$ and $\mathrm{SVM}_{\mathrm{D}^{\prime} \mathrm{Amico}}{ }^{\circ}$ 
prediction of PSA failure in 158 consecutive cases. These nomograms can be competitive to the available clinical nomograms such as the D'Amico risk scheme [7], the CAPRA score [8], and the Stephenson nomogram [6].

Our study improved the predictive performance by applying a robust SVM analysis with MR and clinicpathologic variables. The SVM produced a 93.3\% SEN, 91.7\% SPE and 92.2\% ACC, showing significantly better performance ability than LR approach. This can be attributed to the fact that SVM classifier depends only on the support vectors, and the classifier function is not influenced by the entire data set. It can progressively learns from misclassified examples and automatically remove the false positives via examination of the distance in the Hilbert space to avoid over-fitting. Second, RBFSVM uses a cost-factor to control for classifier complexity and the prediction accuracy which would decrease in case of small cost-factor. Although the data presented in this paper were based on an optimal cost-factor of 2.934, we tested a range of different cost-factor values from 0.1 to 50 and found very similar results, suggesting its good reproducibility. Third, different to the nomograms reported by Poulakis [9], Fuchsjäger [32] and Park [33], our study included more new imaging markers, i.e., DCE-type, Dmax, ADCs and MR T-stage, which may contributed to the improvement of predictive performance. This is true as the performance of clinical D'Amico scheme can be efficiently increased by adding these imaging markers. The imaging contribution in $\mathrm{PCa} \mathrm{BCR}$ predicting can be explained that, the newly proposed imaging markers, i.e., ADCs, DCE type, MR T-stage, might indicate the postoperative histopathological features of $\mathrm{PCa}$ more accurately than conventional clinical variables. This is true as the quantitative MR imaging parameters, e.g., DCE-derived Ktrans, DWI-derived ADCs and/or Dapp, had been identified to be highly associated with the localized cancer aggressiveness and prognosis in recent clinical studies [16-18, 33, 35]. Additionally, we found that, among all imaging markers, only tumor ADCs was independently related to 3-years BCR, suggesting its prognostic value in the prediction of clinical outcome in PCa. The overall ACC for ADCs to differentiate low/ intermediate- from high-grade $\mathrm{PCa}$ is $64.9 \%$, partly consistent with previous reports $[16,18,36]$. And our mp-MRI produced high accuracy for detecting surgical ECE (81.9\%) and SVI (95.1\%). This result confirms strongly the thesis that MR findings can complement the clinical nomogram for the improvement of the predictive performance. The nomogram by integrating MR findings and preoperative clinical variables such as D'Amico risk score is noninvasive and prospective, which could be readily applied by clinicians and allows for immediate identification of the variables accounted for BCR before the RP.

An important limitation is the relatively small sample size in this preliminary study. Thus, future work should consider validating the accuracy of our classifier with an independent larger sample of patients. Second, part of input variables, e.g., DCE-type, MR visibility and MR T-stage, was generally interviewed by individual radiologists, the results of which, to some extent, depending on radiologist's experience. Deviation is unavoidable in spite of many efforts to make. Thus, the findings under this calculation should be considered with caution. Finally, as this study used data from a single medical center, it remains unclear to what extent differences in acquisition protocol or scanners or patient cohort affect the accuracy of the classifier.

\section{CONCLUSIONS}

A SVM approach was more accurate than the classical LR analysis with the same input variables in the prediction of 3-years BCR in PCa after RP. Additionally, MR parameters can efficiently improve the predictive performance of popularized D'Amico scheme. This confirms the thesis that MR findings can well complement the preoperative clinic-pathologic variables to improve existing outcome prediction tools in $\mathrm{PCa}$. As this machine learning approach is benefited with no user input, optimal reproducibility, faster post-processing times, and the ability to use the full potential of the combined clinical and diagnostic variables, which could be readily applied by clinicians to preoperatively assess the therapeutic risk.

\section{MATERIALS AND METHODS}

\section{Patients}

Our local institutional review board approved and waived the informed consent requirement for this retrospective study. Between January 2009 and February 2013, 295 consecutive patients with biopsy confirmed PCa underwent prostatic MRI before RP. Patients received postoperatively immediate adjuvant hormone or radiation therapy before a documented decease recurrence $(n=$ $23,7.8 \%$ ), patients who were lost from follow-up ( $n=$ $19,6.4 \%$ ), and patients failed to receive a standardized MR examination $(n=21,7.1 \%)$ or underwent the MR examination from outside institutions $(n=27,9.2 \%)$ were excluded from the study. Thus, 205 patients were eligible for clinical evaluation. No patient received any neoadjuvant therapy. The median time interval between the MR examination and prostatectomy was 12 days (range, 5-17 days).

\section{Prostatic MR examination}

All imaging examinations were performed with 3.0T MR scanners (Trio and Verio Tim; Siemens, Erlangen, 
Germany) and pelvic phased-array coils. As per the standard prostatic MRI at our institution, the images obtained included transverse $\mathrm{T}_{1}$-weighted turbo spin-echo (TSE) images (TR/TE, 700/14 ms; section thickness, 3.5 $\mathrm{mm}$; intersection gap, $0.3 \mathrm{~mm}$; field of view, $25 \mathrm{~cm}$; and matrix, $384 \times 336$ ) and transverse, coronal, and sagittal T2-weighted TSE images (TR/TE, 6000/124 ms; section thickness, $3.5 \mathrm{~mm}$; intersection gap, $0.3 \mathrm{~mm}$; field of view, $25 \mathrm{~cm}$; and matrix, $384 \times 336$ ) of the prostate and seminal vesicles. Then, single-shot echo-planar imaging (TR/TE, 6800/98 ms; field of view, $25 \mathrm{~cm}$; matrix, $192 \times$ 130; section thickness, $3.5 \mathrm{~mm}$; intersection gap, $0.3 \mathrm{~mm}$; and a parallel imaging factor of 2) was performed with diffusion-module and fat suppression pulses. Diffusion in three directions was measured by using $b$ values of $0,50,150,300,600$, and $1000 \mathrm{~s} / \mathrm{mm}^{2}$. After a routine MR examination, a T1-weighted gradient recalled echo sequence was prescribed to acquire DCE-MR imaging data. This protocol was performed with parameters as follows: TR/TE, 3.8/1.8; flip angle, $12^{\circ}$; field of view, $36 \mathrm{~cm}$; matrix, $384 \times 384$; slice thickness, $3.5 \mathrm{~mm}$; intersection gap, $0.3 \mathrm{~mm}$ ). After two acquisitions, a bolus of Gd-diethylenetriaminepenta-acetic acid (Gd-DTPA $0.05 \mathrm{mmol} / \mathrm{kg}$; Magnevist, Bayer AG, Berlin, Germany) was injected at a rate of $2.5 \mathrm{ml} / \mathrm{s}$ through a 20 -gauge antecubitalintravenous line. Bolus injection was performed with a MR-compatible power injector (Spectris; Medrad, Pittsburgh, PA) followed by a $15-\mathrm{ml}$ saline flush. The DCE-MR imaging was continued for 5.0 minutes after the Gd-DTPA injection.

\section{Imaging analysis}

Imaging analysis was completed independently by two experienced radiologists (Y.Z. and X.W. with 6 and more than 20 years of experience in reading prostate MR imaging). Because this study is to determine whether the noninvasive mp-MRI has prospective value in predicting BCR before the RP operation, any histologicradiologic correlation was prohibited during the MR imaging analysis. In this procedure, acquisition date and participant identification were removed from all images. The investigators were blinded to all clinical information. The mp-MR images from axial $\mathrm{T}_{2} \mathrm{WI}$, DWI and DCE were registered by expending a DICOM-tags metrics on a computer-aided platform (FireVoxel; Center for Advanced Imaging Innovation and Research $\left[\mathrm{CAI}^{2} \mathrm{R}\right]$, New York University School of Medicine, New York, NY). The fusion images were displayed simultaneously and explored slice by slice to facilitate lesion discovery and region of interest (ROI) drawing. In each prostate, the radiologists first identified the most suspicious cancer lesion (leading lesion) on peripheral zone (PZ) and/or transition zone (TZ). The observation featured with largest lesion size, and/or dominantly low signal intensity (SI) on apparent diffusion coefficient (ADC) maps, and/or suspected extracapsular extension (ECE), and/or suspected seminal vesicle invasion (SVI), was defined as the leading lesion (Figure 1). Images was rated independently by the two reviewers according to the guidelines of European Society of Urogenital Radiology (ESUR) [37]. The T WI, DWI and DCE images were scored (1-5) using the Prostate Imaging and Reporting and Data System (PI-RADS) v2 [2], a summed score of all three MR sequences $\left(\mathrm{T}_{2} \mathrm{WI}\right.$, DWI, and DCE) was then calculated for the leading lesion in each patient. Additionally, the following imaging characteristics were summarized: 1) tumor location (PZ or TZ); 2) tumor max diameter (Dmax); 3) tumor is MR-visible or not; 4) tumor ADCs; 5) the DCE type; 6) the presence or absence of ECE (MR stage T3a); 7) the presence or absence of SVI (MR stage T3b); 8) the presence or absence of local lymph node (LN) invasion; and 9) the presence or absence of local bone metastasis. Leading lesion boundaries were determined by manually outlining the regions of interest (ROIs) on fusion $\mathrm{T}_{2} \mathrm{WI}$ and high b-value DWI slice-by-slice. Whole-lesion mean ADC value was measured by expending a monoexponential fitting model. DCE-MRI was classified as three types based on the ESUR guidelines: type 1: slow wash-in and slow wash-out; type 2: fast wash-in and slow wash-out; and type 3: fast wash-in and fast wash-out. The presence of ECE was defined as an infiltration of the periprostatic fat, irregular bulging associated with disruption of the capsule, focal thickening, capsular retraction, and peri-capsular spicula. The features of SVI include focal or diffuse low $\mathrm{T}_{2}$ SI and/or abnormal contrast enhancement within and/or along the seminal vesicle, restricted diffusion, obliteration of the angle between the base of the prostate and the seminal vesicle, and demonstration of direct tumor extension from the base of the prostate into and around the seminal vesicle (Figure 2). Regarding LN invasion, the LNs over $8 \mathrm{~mm}$ in short axis dimension are regarded as suspicious. Nodal groups including common femoral, obturator, external iliac, internal iliac, common iliac, pararectal, presacral, and paracaval, and paraaortic to the level of the aortic bifurcation were evaluated. Local bone metastasis was suspected if focal foci with low SI on $\mathrm{T}_{1} \mathrm{WI}$, high SI on $\mathrm{T}_{2} \mathrm{WI}$ or DWI on pelvic bone. During the image interpretation, any inter-reader disagreement in classifying MR findings of the lesion was discussed until consensus reached.

\section{Histopathology}

The results of the needle biopsy were recorded from the retrospective database, into which they were entered before RP. All prostate biopsies were obtained using TRUS guidance and were reviewed by dedicated urological pathologists (H.L. and M.B., with 10 years of experience in genitourinary pathology). Each core containing cancer was assigned a primary and a secondary Gleason grade. An overall biopsy Gleason score (GS) was 
given to each case by identifying the core with the highest Gleason grade. The total number of cores obtained, the number of cores containing cancer, and the percent of biopsy cores involved with cancer (positive for cancer) were recorded. The prostatic specimens after RP were prepared as previously described [16] and examined by the institutional pathology department. The location and extent of invasive tumors were identified and precisely mapped in each section. An overall pathological GS was assigned to the whole gland. Pathological variables included in the study were the tumor number (small tumors with a volume of less than $0.5 \mathrm{~cm}^{3}$ were excluded), location, tumor volume and volume percent, primary and secondary GS, cancer invasion on the surgical margin, the presence or absence of ECE, SVI, perineural invasion, and the local LN invasion. Pathological stage of the cancer was assessed according to the 2005 International Society of Urological Pathology Modified Gleason Grading System [38].

\section{Follow-up}

The follow-up included measurements of serum PSA level and a digital examination of rectum (DRE) every 3 months for the first year after RP, at 6-month intervals for the next 2 years, and annually thereafter. In accordance with the European Association of Urology (EAU) guidelines for $\mathrm{PCa}$, disease progression was defined as a serum PSA level that failed to decrease to undetectable levels after surgery, or an undetectable PSA level after surgery with a subsequent detectable PSA level that increased on two or more laboratory determinations, or secondary therapy or clinical recurrence [2]. In this study, a detectable level of PSA (i.e., $\geq 0.2 \mathrm{ng} / \mathrm{mL}$ ) after surgery was defined as BCR.

\section{Model development}

To predict the probabilities of PCa BCR associated with the variables, firstly, we constructed two risk predictive models based on SVM and multivariate LR analysis. Patient age ( $<60$ ys and $\geq 60$ ys of age) and seven MR imaging parameters, including the PI-RADS score, tumor location (PZ or TZ), tumor max diameter (Dmax), MR-visible or not, tumor ADCs, tumor DCE type (type $1+2$ or type 3 ), and tumor MR T-stage ( $<$ T3a, T3a, or $>\mathrm{T} 3 \mathrm{a})$, were included as input data for SVM and LR model. We did not include LN invasion and bone metastasis as the predictive variables because we observed a very few cases with lymph node metastasis ( $n$ $=3)$ and bone metastasis $(n=0)$ in this cohort of patients. Secondly, a SVM model based on a popularized D'Amico risk scheme [7] were constructed using the following preoperative clinic-pathologic variables: patient age, the first incorporated preoperative PSA level $(<10 \mathrm{ng} / \mathrm{ml}, 10$
$20 \mathrm{ng} / \mathrm{ml}$ and $>20 \mathrm{ng} / \mathrm{ml})$, needle-biopsy GS $(3+3,3+4$, $4+3$, and $\geq 4+4$ ), and DRE and/or TRUS-based clinical TNM stage. In order to investigate whether the addition of MR variables can further improve the performance ability of the D'Amico model in predicting PCa BCR, a modified D'Amico model was constructed by adding the MR variables.

For the multiple LR model, we determined probabilities (Pi) of PCa BCR by using a backward stepwise approach as follows:

$$
P i=\frac{1}{1+e^{-k}} \text { [eq.1], }
$$$$
k=\sum_{1}^{i} B i X i \text { [eq.2], where } \mathrm{Xi} \text { is the predictive }
$$
variables and $\mathrm{Bi}$ is the regression coefficients determined by the multiple logistic regression analysis.

SVM is a supervised machine learning technique used for classification and regression analysis. SVM algorithm tries to construct an optimal separating hyperplane that maximizes the margin, where the margin is the largest distance to the nearest training data point of any class. Unlike the traditional artificial neural networks (ANNs), the SVM does not have to undertake a trial and error parameter decision process, while determines optimal performance conditions automatically if the kernel type is set. In this study, SVM with radial basis function (RBF) kernel was applied to resolve the two class problems, BCR or BCR-free. A RBF kernel, K, maps the original data with the kernel function as:

$\mathrm{K}(\boldsymbol{x})=\exp \left(-\mathrm{g} \| \boldsymbol{x}\right.$ - $\left.\mathrm{t} \|^{2}\right)$ [eq.3], where $x$ and t are two feature vectors, and Gamma (g) controls the shape of the decision hyperplane. As in this relatively small patient groups, SVM models were developed and validated using a five-fold cross-validation method that maintained the best compromise between computational cost and reliable estimates. The dataset was randomly divided into 5 mutually exclusively subsets of approximately equal size $(n=41)$, in which 4 subsets were used as training set, and the last subset was used as validation set. The sensitivity and specificity values were calculated for each test fold on the trained model of the other four folds. The performance of each iteration was calculated as the average of the performance values of these five folds. This procedure was repeated for five times, so each subset was used once for validation. As the direct output value of the SVM does not show probabilities of PCa BCR, we converted their output values to the probabilities $(P i)$ by applying a sigmoid function as follows:

$$
P i=\frac{1}{1+e^{-\mathrm{x}}} \text { [eq.4], where } x \text { is the output value of }
$$
SVM analysis. The value of $P i$ indicates the probabilities that the patient has PCa BCR. 


\section{Statistical analysis}

The performance of the LR, SVM, D'Amico and modified D'Amico model was evaluated using a receiver operating curve (ROC) analysis to predict the probabilities of PCa BCR. The ROC curves were estimated using a MedCalc statistical software (version 8.2.0.1, MedCalc Software, Mariakerke, Belgium). The performance parameters including the areas under the ROC curve (Az), sensitivity (SEN), Specificity (SPE) and accuracy (ACC) were reported. Pairwise comparison of the ROC curves was performed. Predicted Kaplan-Meier curves from four nomograms were created and compared to patients' true Kaplan-Meier curves using a Kaplan-Meier analysis by SPSS (Version 22.0, Chicago, IL, USA). A multivariable Cox regression analysis was recruited to determine the independent indictors of $\mathrm{PCa} \mathrm{BCR}$, and hazard ratio (HR) was reported. A p value less than 0.05 was considered to indicate a statistically significant difference.

\section{CONFLICTS OF INTEREST}

All authors declare that we have no financial and personal relationships with other people or organizations that can inappropriately influence our work, there is no professional or other personal interest of any nature or kind in any product, service and/or company that could be construed as influencing the position presented in, or the review of the manuscript.

\section{REFERENCES}

1. Heidenreich A, Bastian PJ, Bellmunt J, Bolla M, Joniau $\mathrm{S}$, van der Kwast $\mathrm{T}$, Mason M, Matveev V, Wiegel T, Zattoni F, Mottet $\mathrm{N}$ and European Association of $\mathrm{U}$. EAU guidelines on prostate cancer. Part II: Treatment of advanced, relapsing, and castration-resistant prostate cancer. European urology. 2014; 65(2):467-479.

2. Heidenreich A, Bastian PJ, Bellmunt J, Bolla M, Joniau $\mathrm{S}$, van der Kwast $\mathrm{T}$, Mason M, Matveev V, Wiegel T, Zattoni F, Mottet N and European Association of U. EAU guidelines on prostate cancer. part 1: screening, diagnosis, and local treatment with curative intent-update 2013. European urology. 2014; 65(1):124-137.

3. Abdollah F, Boorjian S, Cozzarini C, Suardi N, Sun M, Fiorino C, di Muzio N, Karakiewicz PI, Montorsi F, Karnes RJ and Briganti A. Survival following biochemical recurrence after radical prostatectomy and adjuvant radiotherapy in patients with prostate cancer: the impact of competing causes of mortality and patient stratification. European urology. 2013; 64(4):557-564.

4. Rigatti P, Suardi N, Briganti A, Da Pozzo LF, Tutolo M, Villa L, Gallina A, Capitanio U, Abdollah F, Scattoni V, Colombo R, Freschi M, Picchio M, Messa C, Guazzoni G and Montorsi F. Pelvic/retroperitoneal salvage lymph node dissection for patients treated with radical prostatectomy with biochemical recurrence and nodal recurrence detected by [11C]choline positron emission tomography/computed tomography. European urology. 2011; 60(5):935-943.

5. Capitanio U, Scattoni V, Freschi M, Briganti A, Salonia A, Gallina A, Colombo R, Karakiewicz PI, Rigatti P and Montorsi F. Radical prostatectomy for incidental (stage $\mathrm{T} 1 \mathrm{a}-\mathrm{T} 1 \mathrm{~b})$ prostate cancer: analysis of predictors for residual disease and biochemical recurrence. European urology. 2008; 54(1):118-125.

6. Stephenson AJ, Scardino PT, Eastham JA, Bianco FJ, Jr., Dotan ZA, Fearn PA and Kattan MW. Preoperative nomogram predicting the 10 -year probability of prostate cancer recurrence after radical prostatectomy. Journal of the National Cancer Institute. 2006; 98(10):715-717.

7. D'Amico AV, Whittington R, Malkowicz SB, Schultz D, Blank K, Broderick GA, Tomaszewski JE, Renshaw AA, Kaplan I, Beard CJ and Wein A. Biochemical outcome after radical prostatectomy, external beam radiation therapy, or interstitial radiation therapy for clinically localized prostate cancer. Jama. 1998; 280(11):969-974.

8. Cooperberg MR, Pasta DJ, Elkin EP, Litwin MS, Latini DM, Du Chane J and Carroll PR. The University of California, San Francisco Cancer of the Prostate Risk Assessment score: a straightforward and reliable preoperative predictor of disease recurrence after radical prostatectomy. The Journal of urology. 2005; 173(6):19381942.

9. Poulakis V, Witzsch U, de Vries R, Emmerlich V, Meves $\mathrm{M}$, Altmannsberger $\mathrm{HM}$ and Becht E. Preoperative neural network using combined magnetic resonance imaging variables, prostate specific antigen, and Gleason score to predict prostate cancer recurrence after radical prostatectomy. European urology. 2004; 46(5):571-578.

10. Lughezzani G, Budaus L, Isbarn H, Sun M, Perrotte P, Haese A, Chun FK, Schlomm T, Steuber T, Heinzer H, Huland H, Montorsi F, Graefen M and Karakiewicz PI. Head-to-head comparison of the three most commonly used preoperative models for prediction of biochemical recurrence after radical prostatectomy. European urology. 2010; 57(4):562-568.

11. Morote J, Del Amo J, Borque A, Ars E, Hernandez C, Herranz F, Arruza A, Llarena R, Planas J, Viso MJ, Palou J, Raventos CX, Tejedor D, Artieda M, Simon L, Martinez A, et al. Improved prediction of biochemical recurrence after radical prostatectomy by genetic polymorphisms. The Journal of urology. 2010; 184(2):506-511.

12. Yakar D, Debats OA, Bomers JG, Schouten MG, Vos PC, van Lin E, Futterer JJ and Barentsz JO. Predictive value of MRI in the localization, staging, volume estimation, assessment of aggressiveness, and guidance of radiotherapy and biopsies in prostate cancer. Journal of magnetic resonance imaging. 2012; 35(1):20-31.

13. Jung SI, Donati OF, Vargas HA, Goldman D, Hricak H and Akin O. Transition zone prostate cancer: incremental value 
of diffusion-weighted endorectal MR imaging in tumor detection and assessment of aggressiveness. Radiology. 2013; 269(2):493-503.

14. Vargas HA, Akin O, Franiel T, Mazaheri Y, Zheng J, Moskowitz C, Udo K, Eastham J and Hricak H. Diffusionweighted endorectal $\mathrm{MR}$ imaging at $3 \mathrm{~T}$ for prostate cancer: tumor detection and assessment of aggressiveness. Radiology. 2011; 259(3):775-784.

15. Soylu FN, Peng Y, Jiang Y, Wang S, Schmid-Tannwald C, Sethi I, Eggener S, Antic T and Oto A. Seminal vesicle invasion in prostate cancer: evaluation by using multiparametric endorectal MR imaging. Radiology. 2013; 267(3):797-806.

16. Wang Q, Li H, Yan X, Wu CJ, Liu XS, Shi HB and Zhang YD. Histogram analysis of diffusion kurtosis magnetic resonance imaging in differentiation of pathologic Gleason grade of prostate cancer. Urologic oncology. 2015; 33(8):337 e315-324.

17. Zhang YD, Wang Q, Wu CJ, Wang XN, Zhang J, Liu H, Liu XS and Shi HB. The histogram analysis of diffusionweighted intravoxel incoherent motion (IVIM) imaging for differentiating the gleason grade of prostate cancer. European radiology. 2015; 25(4):994-1004.

18. Hambrock T, Somford DM, Huisman HJ, van Oort IM, Witjes JA, Hulsbergen-van de Kaa CA, Scheenen T and Barentsz JO. Relationship between apparent diffusion coefficients at 3.0-T MR imaging and Gleason grade in peripheral zone prostate cancer. Radiology. 2011; 259(2):453-461.

19. Rosenkrantz AB, Kim S, Lim RP, Hindman N, Deng FM, Babb JS and Taneja SS. Prostate cancer localization using multiparametric MR imaging: comparison of Prostate Imaging Reporting and Data System (PI-RADS) and Likert scales. Radiology. 2013; 269(2):482-492.

20. Lee HJ, Hwang SI, Han SM, Park SH, Kim SH, Cho JY, Seong CG and Choe G. Image-based clinical decision support for transrectal ultrasound in the diagnosis of prostate cancer: comparison of multiple logistic regression, artificial neural network, and support vector machine. European radiology. 2010; 20(6):1476-1484.

21. Galinsky KJ, Bhatia G, Loh PR, Georgiev S, Mukherjee S, Patterson NJ and Price AL. Fast Principal-Component Analysis Reveals Convergent Evolution of ADH1B in Europe and East Asia. American journal of human genetics. 2016; 98(3):456-472.

22. Li B, Calvet A, Casamayou-Boucau Y and Ryder AG. Kernel principal component analysis residual diagnosis (KPCARD): An automated method for cosmic ray artifact removal in Raman spectra. Analytica chimica acta. 2016; 913:111-120.

23. Wang S, Phillips P, Yang J, Sun P and Zhang Y. Magnetic resonance brain classification by a novel binary particle swarm optimization with mutation and time-varying acceleration coefficients. Biomedizinische Technik Biomedical engineering. 2016.
24. Larroza A, Moratal D, Paredes-Sanchez A, Soria-Olivas E, Chust ML, Arribas LA and Arana E. Support vector machine classification of brain metastasis and radiation necrosis based on texture analysis in MRI. Journal of magnetic resonance imaging. 2015; 42(5):1362-1368.

25. Wang Z. Support vector machine learning-based cerebral blood flow quantification for arterial spin labeling MRI. Human brain mapping. 2014; 35(7):2869-2875.

26. Bevilacqua V, Pannarale P, Abbrescia M, Cava C, Paradiso A and Tommasi S. Comparison of data-merging methods with SVM attribute selection and classification in breast cancer gene expression. BMC bioinformatics. 2012; 13 Suppl 7:S9.

27. Emblem KE, Due-Tonnessen P, Hald JK, Bjornerud A, Pinho MC, Scheie D, Schad LR, Meling TR and Zoellner FG. Machine learning in preoperative glioma MRI: survival associations by perfusion-based support vector machine outperforms traditional MRI. Journal of magnetic resonance imaging. 2014; 40(1):47-54.

28. Mourao-Miranda J, Reinders AA, Rocha-Rego V, Lappin J, Rondina J, Morgan C, Morgan KD, Fearon P, Jones PB, Doody GA, Murray RM, Kapur S and Dazzan P. Individualized prediction of illness course at the first psychotic episode: a support vector machine MRI study. Psychological medicine. 2012; 42(5):1037-1047.

29. Peng Y, Jiang Y, Yang C, Brown JB, Antic T, Sethi I, Schmid-Tannwald C, Giger ML, Eggener SE and Oto A. Quantitative analysis of multiparametric prostate MR images: differentiation between prostate cancer and normal tissue and correlation with Gleason score-a computeraided diagnosis development study. Radiology. 2013; 267(3):787-796.

30. Sung YS, Kwon HJ, Park BW, Cho G, Lee CK, Cho KS and Kim JK. Prostate cancer detection on dynamic contrast-enhanced MRI: computer-aided diagnosis versus single perfusion parameter maps. AJR American journal of roentgenology. 2011; 197(5):1122-1129.

31. Poulakis V, Witzsch U, De Vries R, Emmerlich V, Meves M, Altmannsberger HM and Becht E. Preoperative neural network using combined magnetic resonance imaging variables, prostate specific antigen and Gleason score to predict prostate cancer stage. The Journal of urology. 2004; 172(4 Pt 1):1306-1310.

32. Fuchsjager MH, Shukla-Dave A, Hricak H, Wang L, Touijer K, Donohue JF, Eastham JA and Kattan MW. Magnetic resonance imaging in the prediction of biochemical recurrence of prostate cancer after radical prostatectomy. BJU international. 2009; 104(3):315-320.

33. Park SY, Kim CK, Park BK, Lee HM and Lee KS. Prediction of biochemical recurrence following radical prostatectomy in men with prostate cancer by diffusionweighted magnetic resonance imaging: initial results. European radiology. 2011; 21(5):1111-1118.

34. Park SY, Oh YT, Jung DC, Cho NH, Choi YD, Rha KH and Hong SJ. Prediction of biochemical recurrence after radical 
prostatectomy with PI-RADS version 2 in prostate cancers: initial results. European radiology. 2016; 26(8):2502-2509.

35. Park JJ, Kim CK, Park SY, Park BK, Lee HM and Cho SW. Prostate cancer: role of pretreatment multiparametric 3-T MRI in predicting biochemical recurrence after radical prostatectomy. AJR American journal of roentgenology. 2014; 202(5):W459-465.

36. Turkbey B, Shah VP, Pang Y, Bernardo M, Xu S, Kruecker J, Locklin J, Baccala AA, Jr., Rastinehad AR, Merino MJ, Shih JH, Wood BJ, Pinto PA and Choyke PL. Is apparent diffusion coefficient associated with clinical risk scores for prostate cancers that are visible on 3-T MR images? Radiology. 2011; 258(2):488-495.
37. Barentsz JO, Richenberg J, Clements R, Choyke P, Verma S, Villeirs G, Rouviere O, Logager V, Futterer JJ and European Society of Urogenital R. ESUR prostate MR guidelines 2012. European radiology. 2012; 22(4):746-757.

38. Epstein JI, Allsbrook WC, Jr., Amin MB, Egevad LL and Committee IG. The 2005 International Society of Urological Pathology (ISUP) Consensus Conference on Gleason Grading of Prostatic Carcinoma. The American journal of surgical pathology. 2005; 29(9):1228-1242. 